\title{
A Simple Scheme for Improved Performance of Fixed Outage Rate Cellular System
}

\author{
${ }^{1}$ Hussein M. Aziz Basi and ${ }^{2}$ M.B.R. Murthy \\ ${ }^{1}$ Faculty of Information Science and Technology, \\ ${ }^{2}$ Faculty of Engineering and Technology, \\ Multimedia University, Jalan Ayer Keroh Lama, 75450 Melaka, Malaysia
}

\begin{abstract}
The traffic characteristic of mobile cellular systems is rather distinct from that of a fixed telephone network. However the system planning and design are still carried out with the tools of conventional traffic theory. In the recent days much work is being done in the performance evaluation of mobile or cellular communication systems in order to develop a system with greater efficiency. The useful parameters to estimate the performance of the system are voice quality, frequency spectrum efficiency and Grade of Service (GOS). The grade of service will be affected due to outage of channels. In this study, a new scheme is proposed to reduce lost calls due to channel outage in the fixed rate outage cellular system. In this proposed scheme the call will never dropped but may be delayed. The system performance is evaluated for different conditions and the results are discussed.
\end{abstract}

Key words: Outage Channel, Delay Probability, Priority Calls, Waiting Time

\section{INTRODUCTION}

A mobile system is a communications network in which at least one of the constituent entities that is used, switches, or a combination of both change locations relative to one another. With the advancements in wireless technology mobile communication is growing rapidly.

In the design of high-capacity cellular systems quality of communication and channel capacity (the number of users that can be supported by the system) are of prime importance. In order to determine the optimal channel loading it is necessary to relate the Grade of Service (GOS) to traffic characteristics. The GOS is defined in several ways ${ }^{[1,2,3]}$. GOS is an indication of blocked calls and delayed calls. The GOS in cellular system is affected not only by the system's traffic but also by co-channel interference.

During a link communication between two subscribers in analog cellular system, the user may experience noise or cross talk or absence of the desired signal resulting in link outage. Even if link outages are of very short duration they may not be individually recognized, but collectively they degrade the system performance ${ }^{[4]}$. In a cellular system, the presence of co-channel interference can cause the signal to noise ratio (C/I or SIR) to drop below a specified threshold level $\varphi$. Such an event is known as an outage. This can cause loss of the communication and affect the grade of service ${ }^{[5,6]}$. Outage probability is one of the most important criteria for evaluating the performance of cellular networks ${ }^{[7]}$. A study of the grade of service degradation due to the outage will be useful in evaluating the system performance. Also, it could help the telecommunications industry in developing more robust systems.

The normal system models are developed on the basis of Markov chain using the M/M/C queue model. A new call comes to the cellular system by arrival rate 1. If there is an available channel in the cell, a call is connected and the channel becomes busy. The call service rate is taken to be me. If there is no available channel the call is blocked. The Erlang's C formula is used to calculate the probability of delay as given by ${ }^{[8]}$ and the formula is:

$P_{D}=C(N, A)=\frac{A^{N}}{N !} \frac{P_{0}}{1-\frac{A}{N}}$

Where $P_{0}=\left[\sum_{i=0}^{N-1} \frac{A^{i}}{i !}+\frac{A^{N}}{(N) !} \frac{1}{1-\frac{A}{N}}\right]^{-1}$

where, $\mathrm{N}$ is the total number of channels available and $\mathrm{A}$ is the traffic intensity given by $\mathrm{A}=(\lambda / \mu)$. Even when the call gets connected if the $C / I$ ratio drops below threshold the channel goes to outage and communication is affected or lost. From the normal working condition the line goes to outage condition, when the call is completed or channel recovers from outage again it becomes available. 


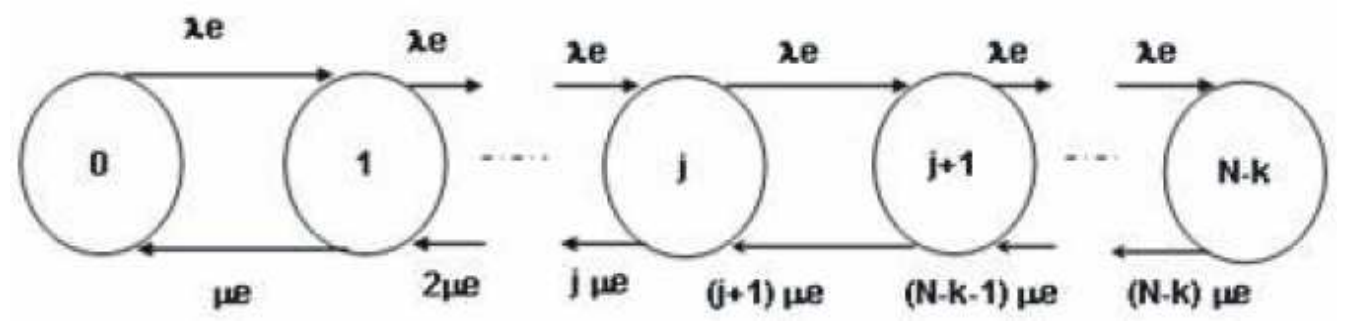

Fig. 1: Markov Chain Model for N-k Channels

In some investigations ${ }^{[6]}$ while estimating the effect of outage it was taken that if there is no available channel the call is blocked or dropped. In this case they did not consider the aspect of the mean waiting time with priority calls. Some researchers have evaluated the performance of mobile systems with the priority concept where in when no line is available the call is queued up. Priority calls are placed in a queue before all non-priority calls but never interrupt a call in progress ${ }^{[9]}$. However they did not consider the concept of outage. In this study both outage and priority concepts are considered in system evaluation.

Present scheme: In this study a new scheme is proposed which consists of a buffer memory of sufficient capacity. The buffer queues up all new incoming calls and outage calls as well. It is assumed that the users are uniformly distributed among the cells and calls are also uniformly and independently generated. The call arrival rate and service rate are taken to be $\lambda$ and $\mu$ respectively in line with the literature. It is assumed that the channel can go into outage at rate $\gamma$ and may recover at the rate $\alpha$. Since we are considering a fixed outage rate model, $\gamma$ will be independent of the state while a will increase with the number of channels in outrage. In other words we can consider $\gamma_{\mathrm{i}}=\gamma, \alpha_{\mathrm{i}}=\mathrm{i} * \alpha$ where $\mathrm{i}$ is the state considered. At any instant of time new calls arrive at rate $\lambda$ and channel outage occurs at rate $\gamma$. Since buffer queues up all calls the effective arrival rate to memory is $\lambda_{\mathrm{e}}=\lambda+\gamma$. Similarly calls are completed at rate $\mathrm{m}$ and outage channels recover at rate $i * \alpha$. Thus the effective rate at which channels are becoming available will be $\mu_{\mathrm{e}}=(\mu$ $+\alpha)$. The effective traffic intensity is taken to be $A_{e}=$ $\left(\lambda_{\mathrm{e}} / \mu_{\mathrm{e}}\right)$ Out of total $\mathrm{N}$ channels with $\mathrm{k}$ channels in outage, the available channels will be N-k. These events are represented by a Markov chain model with $\mathrm{N}-\mathrm{k}$ channels in Fig. 1.

Using the effective rate $\left[\lambda_{\mathrm{e}}=\lambda+\gamma\right]$ instead of $\lambda$, $\left[\mu_{\mathrm{e}}=(\mu+\alpha)\right]$ instead of $\mathrm{m}$ and number of channels $(\mathrm{N}-$ $\mathrm{k}$ ) instead of $\mathrm{N}$ in the equation (1), we get modified Erlang's $\mathrm{C}$ formula giving the probability of delay in the proposed model as:

$$
P_{D}=C\left(N-k, A_{e}\right)=\frac{A_{e}^{N-k}}{(N-k) !} \frac{P_{0}}{1-\frac{A_{e}}{(N-k)}}
$$

Where $P_{0}=\left[\sum_{i=0}^{N-k-1} \frac{A e^{i}}{i !}+\frac{A e^{N-k}}{(N-k) !} \frac{1}{1-\frac{A e}{N-k}}\right]^{-1}$

When there are no channels in outage $\mathrm{k}=0$ and the results obtained using equation (2) will be same as those with equation (1).

For the calculation of mean waiting time of priority and non-priority calls the relations of ${ }^{[3]}$ are used with $\mathrm{N}$ replaced by N-k. The modified relations will be:

$$
\begin{aligned}
\mathrm{WT}_{1} & =\frac{\left(\mathrm{P}_{\mathrm{D}} * \mathrm{~d}\right)}{(\mathrm{N}-\mathrm{k})(1-\mathrm{p} \rho)} \\
\mathrm{WT}_{2} & =\frac{\left(\mathrm{P}_{\mathrm{D}} * \mathrm{~d}\right)}{(\mathrm{N}-\mathrm{k})(1-\mathrm{p} \rho)(1-\rho)}=\frac{\mathrm{WT}_{1}}{1-\rho}
\end{aligned}
$$

$\mathrm{WT}_{1}$ and $\mathrm{WT}_{2}$ are the waiting time for calls with priority and non-priority respectively. $\mathrm{D}$ is the average call duration, $\rho$ is overall system load and $p$ is the priority proportion. The load due to priority calls will be $\rho \times \mathrm{p}^{[3]}$. $\mathrm{P}_{\mathrm{D}}$ is the probability of delay. The probability of delay calculated according to equation (2) is used in the evaluation of the waiting time.

These relations indicate that the normal calls have to wait longer than priority calls.

\section{RESULTS AND DISCUSSION}

The values of probability of delay and waiting times are calculated for priority and non-priority is evaluated using the proposed model for several conditions and are presented in Fig. 2 and 3. The number of channels used for the calculation is 100 . The traffic is assumed to be 500 calls/hour. From the results we find that the probability of delay $P_{D}$ increases with increase in outage channels.

Further we notice that $P_{D}$ depends on traffic intensity. Increase in traffic intensity causes higher probability of delay PD. It is observed that the probability of delay varies linearly for lower values of $\mathrm{k}$. For higher values of $\mathrm{k}$ the variation is exponential. When priority is introduced (Fig. 3) the waiting time for priority calls is less than that of non priority calls and their variation is in line with those reported in literature. 
Table 1: Mean Waiting Time for Priority and Non-Priority Calls for 500 Call Where $N=100 A_{e}=40$ and $\alpha=0.5$

\begin{tabular}{|c|c|c|c|c|c|c|c|}
\hline & $\mathrm{K}$ & PD & 0.1 & 0.2 & 0.3 & 0.4 & $\begin{array}{ll}0.5 \\
\end{array}$ \\
\hline WT1 & 0 & $1.17 \mathrm{E}-02$ & 1.77E-04 & 1.77E-04 & $1.78 \mathrm{E}-04$ & 1.79E-04 & $1.80 \mathrm{E}-04$ \\
\hline WT2 & & & $8.42 \mathrm{E}-02$ & $1.85 \mathrm{E}-04$ & $1.86 \mathrm{E}-04$ & $1.87 \mathrm{E}-04$ & $1.87 \mathrm{E}-04$ \\
\hline WT1 & 10 & $3.05 \mathrm{E}-02$ & $5.11 \mathrm{E}-04$ & 5.13E-04 & $5.15 \mathrm{E}-04$ & $5.18 \mathrm{E}-04$ & $5.20 \mathrm{E}-04$ \\
\hline WT2 & & & 5.33E-04 & $5.36 \mathrm{E}-04$ & $5.38 \mathrm{E}-04$ & $5.40 \mathrm{E}-04$ & $5.42 \mathrm{E}-04$ \\
\hline WT1 & 20 & 7.34E-02 & $1.38 \mathrm{E}-03$ & $1.39 \mathrm{E}-03$ & $1.39 \mathrm{E}-03$ & $1.40 \mathrm{E}-03$ & $1.41 \mathrm{E}-03$ \\
\hline WT2 & & & $1.44 \mathrm{E}-03$ & $1.45 \mathrm{E}-03$ & $1.46 \mathrm{E}-03$ & $1.46 \mathrm{E}-03$ & $1.47 \mathrm{E}-03$ \\
\hline WT1 & 30 & $1.63 \mathrm{E}-01$ & $3.50 \mathrm{E}-03$ & $3.52 \mathrm{E}-03$ & 3.53E-03 & $3.55 \mathrm{E}-03$ & $3.56 \mathrm{E}-03$ \\
\hline WT2 & & & $3.66 \mathrm{E}-03$ & $3.67 \mathrm{E}-03$ & $3.69 \mathrm{E}-03$ & $3.70 \mathrm{E}-03$ & $3.72 \mathrm{E}-03$ \\
\hline WT1 & 40 & 3.33E-01 & $8.36 \mathrm{E}-03$ & $8.40 \mathrm{E}-03$ & $8.43 \mathrm{E}-03$ & 8.47E-03 & $8.50 \mathrm{E}-03$ \\
\hline WT2 & & & $8.73 \mathrm{E}-03$ & $8.76 \mathrm{E}-03$ & $8.80 \mathrm{E}-03$ & $8.84 \mathrm{E}-03$ & $8.87 \mathrm{E}-03$ \\
\hline
\end{tabular}

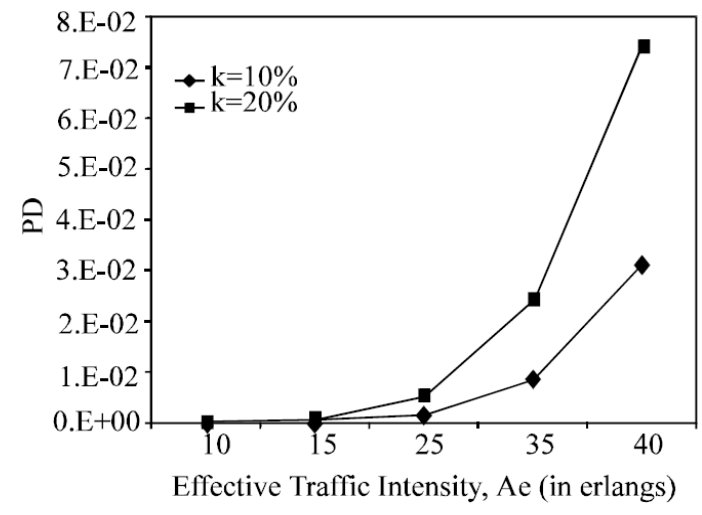

Fig. 2: Probability of Delay Versus Effective Traffic Intensity for Different Outage Load where $\mathrm{N}=100$ and $\gamma=0.00664$

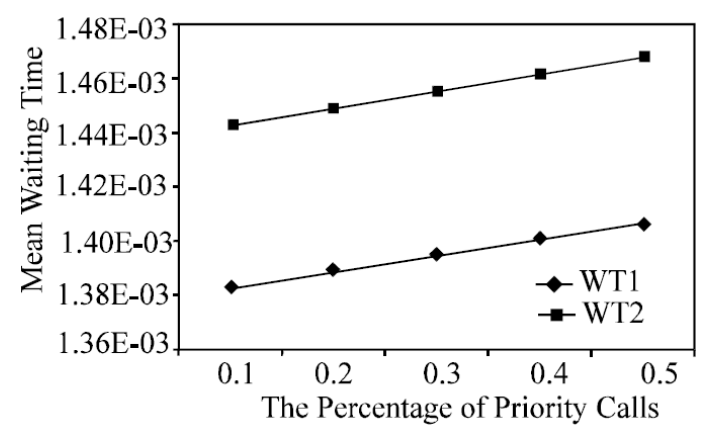

Fig. 3: The Mean Waiting Time for Priority Calls for 100 Channels where $A_{e}=40, \alpha=0.5$ and the Outage Channel 20\%

This is the situation of interest in a mobile channel system with priority. This helps in understanding the delay of calls with outage consideration under various traffic conditions. This also helps to decide the buffer size.

The mean waiting times of priority and nonpriority calls for several conditions estimated using the proposed model are given in Table 1. It is observed that for any load condition the delay is more for non-priority call than that of priority call. Since the outage calls are queued along with normal arriving calls into the buffer, as the number of outage channels increase the waiting times also increase. With the present scheme, due to the introduction of buffer, there may be a marginal increase in delay but no call is lost. In other words the probability of blocking is zero with the proposed method.

\section{CONCLUSION}

A new scheme is proposed for reducing the drop of calls due to channel non-availability or outage channels for the fixed outage rate system. This improves the GOS of the system. The system is tested for different load conditions.

\section{REFERENCES}

1. Hong, H. H., R. Malhamd and Gerald Chen, 1991. Traffic Engineering of Trunked Land Mobile Radio Dispatch System: IEEE; pp. 251-256.

2. Forman, G. H. and J. Zahorjan, 1994. The Challenges of Mobile Computing. Computer Science and Engineering, University of Washington, pp. 1-16.

3. Barcelo, F.,V. Casares and J. Paradells, 1996. M/D/C queue with priority: Application to trunked mobile radio systems. Electron. Lett., 32: 1644-1645.

4. Caini, C., G. Immovilli and M. L. Merani, 1992. Outage Probability for Cellular Mobile Radio Systems: Simplified Analytical Evaluation and Simulation Results. Electron. Lett., 28: 669-671.

5. Annamalai, A. C., Tellambura and V. K. Bhargava, 2001. Simple and Accurate Methods for Outage Analysis in Cellular Mobile Radio System. A Unified Approach. IEEE Transactions on Communications. 49: 303-308.

6. Aguirre, A., D. Munoz, C. Molina and K. Basu, 1998. Outage-GOS Relationship in cellular Systems. IEEE Communications Letters. 2: 5-7.

7. Mazen O Hasna, Mohamed-Slim Alouini and Marvin K. Simon, 2001. Effect of Fading Correlation on the Outage Probability of Cellular Mobile Radio System. IEEE 17941798.

8. Yacoub, M. D., 1993. Foundations of Mobile Radio Engineering. CRC press, Inc.

9. Barcelo, F. and J. Paradells, 2000. Performance Evaluation of Public Access Mobile Radio (PAMR) Systems with Priority calls. IEEE, 979-983. 